\title{
Colitis ulcerosa
}

\author{
Eduardo Chávez C. ${ }^{1}$; Sergio Ceresa O. ${ }^{\mathrm{t}}$; Maureen Rosse] G. ${ }^{1}$; \\ Jorge Las Heras $\mathrm{B}^{2}$
}

Ulcerative colitis

\begin{abstract}
Ulcerative colitis in children has been seldom described in Chile. The cases of a 14 year old girl and a 10 vear old boy with this disease are presented. Both had diarrhea for more than two months - which was continuous in the first case and intermitent in the other one-, bloody stools, weight loss, anemia and abdominal pain. Bacteriological and parasitological examination of stools were negative. Diagnosis of ulcerative colitis was based on barium enema. wich showed mucosal ulceration and loss of the normal hastral pattern, rectosigmoidoscopy, that revealed hyperemia, friability and erosions of the correspondig segments of intestinal mucosa, and on histological examination of multiple mucosal biopsies, which disclosed crypt abscess, distorted crypi pattern, inflammation of the lamina propia and decreased number of goblet celks. Both cases were treated with salazosulfapyridine with satisfactory response.
\end{abstract}

(Key words: ulicerative colitis, diagnosis, treatment, salazosulfapvridine,)

La colitis ulcerosa es una enfermedad inflamatoria crónica de la mucosa de colon y recto de etiología y patogenia desconocidas. Clínicamente se caracteriza por rectorragias y diarrea recurrente ${ }^{1,2}$. Las características histopatológicas de la enfermedad incluyen distorsión de las criptas, infiltrado linfoplasmocitario y polimorfonuclear de la lámina propia y abscesos de las criptas. La poca frecuencia con que el problema se ha descrito en nuestro medio ${ }^{3-7}$ justifica la presen. tación de estos dos pacientes que consultaron en nuestra unidad, con el propósito de estimular el interés y facilitar el reconocimiento de otros casos de la enfermedad.

\section{Casos Clínicos}

1. Niña de 14 años de edad, ingresó el 20 de diciem. bre de 1983, đespués de dos meses de evolución con deposicjones líquidas intermitentes, progesivamente enterocólicas, rectorragia, dolores cólicos abdominales, decaimiento, palidez, compromiso severo del estado de nutrición y antecedentes ae contacto con tuberculosis, por lo que habia recibido tratamiento específico a los 3 años de edad. Estaba decaída, enfla-

1. Unidad de Gastroenterología, Servicio de Pediatría, Hospital Clínico San Borja-Ariarán.

2. Unidad de Anatomía Patológica, Hospital Clinico San Borja-Arriarán. quecida, pesaba 30 kilos -percentil 5 del Centro de Estadísticas de Salud de EUA (NCHS)-, talla $156 \mathrm{~cm}$ -percentil 25 NCHS-, peso para la talla percentil $<5$ NCHS, palidez de piel y de mucosas, distersión abdominal, dolor a la palpación y resistencia muscular en flanco derecho. Extremidades enflaquecidas. Hematoct ito $27 \%$; leucocitos $6.400 \times \mathrm{mm}^{3}, 49 \%$ baciliformes; velocidad de eritrosedimentación (VHS) $50 \mathrm{~mm}$ primera hora, albúmina plasmática $1,79 \mathrm{~g} / \mathrm{dl}$, Reacciones de aglutinación para fiebre tifoidea, coprocultivos, exámenes parasitológioos seríados de deposiciones, búsqueda de bacilos de Koch en orina, expectoración y contenido gástrico dieron resultados negativos. Ecotomografía abdominal y tadiografías de tórax normales. En la radiogtafía de abdomen simple se registraron signos de distensión de asas, niveles líquidos y colon de paredes lisas. En la rectoscopja, b mucosa se veía enrojecida friable, con mucosidades y úlceras rectales. El estudio histológico de la biopsia rectal mostró dilataciones quísticas y abscesos de ths criptas e infiltrado linfoplasmocitario de la lámina propia. El enema baritado mostró que había pequeñas ulceraciones y pérdida de haustras en el colon descendente Con estos antecedentes, que apoyaban el diagnóstico de colitis ulcerosa, se inició tratamiento con salazosulfapiridina por vía oral, hidrocortisona por vja parenteral, y luego, prednisona oral, enemas de retención con hidrocortisona y apoyo nutricional parenteral. La evolución inicial fue tórpida, con mayor descenso de peso e hipoalbuminemia, complicándose con bronconeumonía y hemorragia digestiva por esofagitis péptica. Sin embargo, mejoró posteriormente, en forma paulatina, se normalizaron sus deposiciones y recuperó el apetito, pudiendo ser dada de alta 93 días después de ingresar, en tratamiento permanente con salazosulfapiridina, Evolucionó sin molestias hasta abril de 1985 , su peso aumentó 
a 38 kilos y su talla a $161 \mathrm{~cm}$, pero entonces dejó de controlarse hasta septiembre de 1986, época en que volvió a consultar por diarrea y rectoragia, después de haber abandonado el tratamiento durante dos meses. Su recuperación se logró nuevamente en diez días, tan pronto se reanudó el uso de salazosulfapiridina. Posteriormente, su evolución ha sido satisfactoria.

2. Varón proveniente de Puerto Montt, de 10 años de edad al consultar en 1987 por sufrir de deposiciones dlarrejcas frecuentes con sangre, a veces nocturnas, dolor abdominal, pujo y tenesmo de 10 meses de duración. Los exámenes bacteriológicos y parasitológicos de deposiciones dieron resultados negativos. Recibió tratamiento con sulfaguanidina sin respues. ta favorable. En agosto de 1987 en la biopsia recta] se encontró evidencia de rectitis inespecífica. Un mes después, dada la persistencia de sus síntomas, se realizó otro tratamiento, también infructuoso, con diyodohjdroxiquinoleína por 15 días, habiendo perdido hasta entonces 5 kilos de peso desde el comienzo de la enfermedad. En enero de 1988 el enema baritado mostró pérdida de haustras e imágenes contrastadas puntiformes en el colon transverso y sigmoides, sugerentes de pequeñas ulceraciones. En marzo de 1988 sus molestias continuaban, estaba enflaquecido, pesaba 32 kilos (p 50 NCHS), su talk era $143 \mathrm{~cm}$ (p 75 NCHS), relación peso/talla (p 10-25 NCHS), abdomen sensible en el flanoo izquierdo. En la tectosigmoidoscopía se observó que la mucose era rígida, poco distensible, hiperémica, frible, con exosiones. La colonoscopín hasta $60 \mathrm{~cm}$ mostró la mucosa también friable, granular y etosionada. En la biopsia rectal habia intensa inflamación, distorsión de las criptas, que tenían abscesos en su interior, y marcada disminución de las células caliciformes (figura 1). El enema baritado mostró granulaciones y ero- siones pequeñas de la mucosa en colon descendente y transver so, compatibles con colitis ulcerosa. El estudio parasitológico de contenido rectal con técnica de polivinil-aleoholformol y los coprocultivos dieron resultados negativos. Con estos antecedentes se inició tratamiento oral con salazosulfapiridina $50 \mathrm{mg} \cdot \mathbf{k g} \cdot$ día, dividido en tes dosis. Se controló en julio de 1988, encontrándose en buenas condiciones generales con aumento de peso a $37 \mathrm{~kg}$, sin diarrea, rectorragia ni tenesmo rectal. En abril de 1989 se demostró remisión de la enfermedad mediante rectoscopja y biopsia rectal. En junio de 1990 estaba en buenas condiciones, asintomático, pesando $53,200 \mathrm{~kg}$ y su talla era $163 \mathrm{~cm}$. Ha continuado con el tratamiento indicado hasta b fecha en forma estricta.

\section{Comentario}

La frecuencia de colitis ulcerosa varia en las distintas zonas geográfjcas. Se ha descrito una incidencia que oscila entre 4,5 y 7,3 en diferentes lugares de Europa y Estados Unidos de América. $\mathrm{La}$ incidencia en nuestro país es desconocida.

Las manifestaciones intestinales de la colitis ulcerosa comprenden: diarreas sanguinolentas, dolor abdominal, tenesmo, anorexia, náuseas y vómitos ${ }^{1,2}$, todos estos síntomas presentes en ambos pacientes de esta serie. Destacan, además, manifestaciones extraintestinales (deshidratación, pérdida de peso, retraso del desarrollo) y signos no especificos de enfermedad inflamatoria

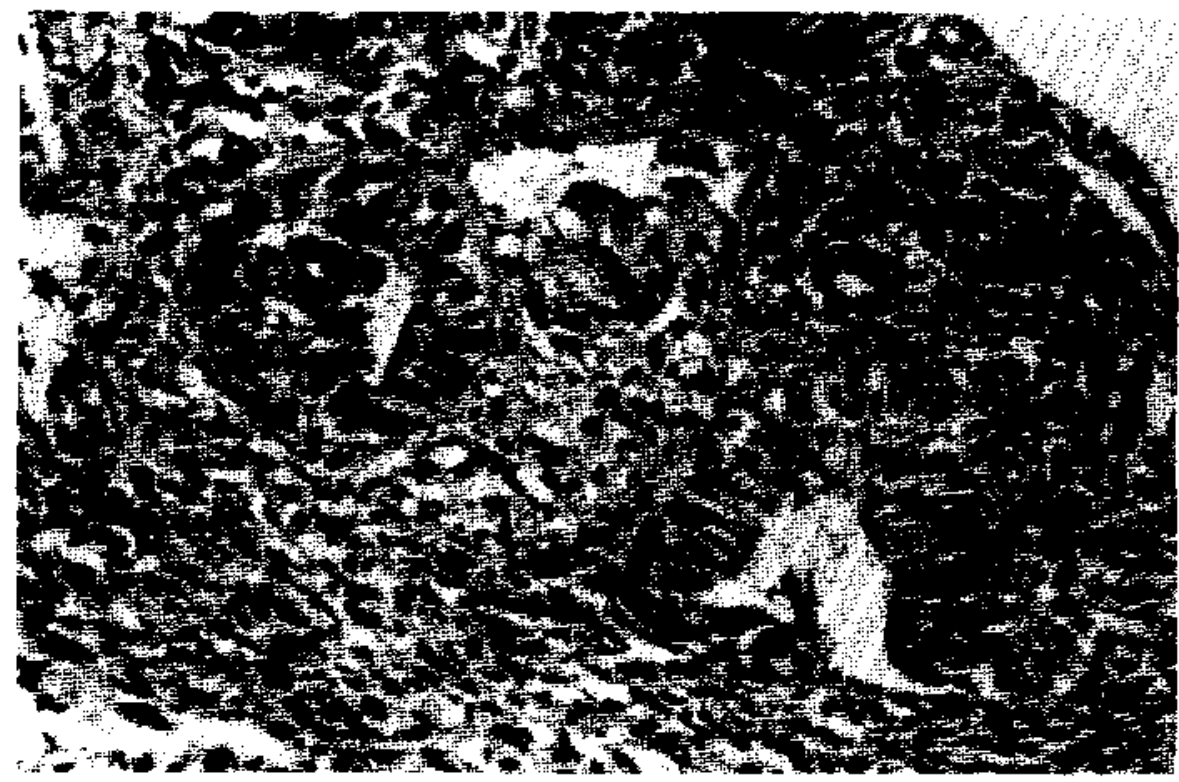

Figura 1: Microfotografía de una biopsia rectal correspondiente al $2^{\circ}$ caso que demuestra abundante infiltrado linfoplasmocitario del estroma vellositario y absceso de una de las criptas. 
(calofríos, fiebre y leucocitosis). El enema baritado demuestra aspecto granular de la mucosa, pérdida de haustras e imágenes sugerentes de ulceraciones superficiales.

En la inspección endoscópica de colon y recto se puede encontrar eritema, edema, pérdida del patrón vascular, mucosa granular y ulceraciones. La biopsia de mucosa colon-rectal en el estado agudo muestra inflamación severa, aumento en el número de linfocitos y de las células plasmáticas de la lámina propia, junto con infiltración focal polimorfonuclear, disminución de células caliciformes y abscesos de las criptas, las cuales pueden estar distorsionadas. Todos estos hallazgos fueron descritos en ambos pacientes, siendo determinantes en el diagnóstico. Algunas de estas características pueden ocurrir en colitis aguda por Salmonella, Shigella, Yersinia enterocolitica, Campylobacter y Entarnoeba histolytica, ${ }^{1,2}$, que deben descartarse mediante los exámenes correspondientes antes de concluir que un niño tiene una colitis ulcerosa crónica no específica. Por esta razón la biopsia debe correlacionarse con hechos clínicos, radiológicos y endoscópicos, El examen endoscópico con biop. sia es esencial para confirmar el diagnóstico y analizar extensión y severidad del proceso inflamatorio.

En el curso clínico de la enfermedad destacan dos modalidades: la colitis crónica continua, que responde escasamente al tratamiento médjco, y la remitente, que es la observada en estos pacientes y se caracteriza por remisiones y exacerbaciones, graves y frecuentes, durante los primeros años de la enfermedad ${ }^{1,2}$.

La extensión de la enfermedad puede comprometer a todo el colon, comportándose como pancolitis o limitándose, de otro modo, a una proctitis ulcerativa o colitis del lado izquierdo ${ }^{2}$. Esta última es la que a nuestro juicio presentaron los dos pacientes que se discuten, siendo necesario, en estos casos, controlar anualmente para pesquisar oportunamente la extensión más allá del ángulo esplénico del colon, pues ésta se asocia a lesiones preneoplásicas, previendo de esta manera el carcinoma de colon ${ }^{8}$.

El tratamiento médico de la colitis ulcerosa comprende el uso de salazosulfapiridina, cuyo componente activo es el ácido 5 aminosalicilico que parece actuar localmente en el colon, disminuyendo la respuesta inflamatoria o interfiriendo con la secreción por inhibición del metabolismo de prostaglandinas ${ }^{1,2}$. Las dosis recomendadas son 50 a $75 \mathrm{mg} \cdot \mathrm{kg} \cdot \mathrm{dia}$, siendo preferible $\infty$ menzar con dosis pequefias y aumentarlas progresivamente para minimizar los efectos indeseables como cefalea, alteraciones gastrointestinales, anemia hemolitica y erupciones cutáneas, que se describen con esta droga.

Los pacientes con colitis ulcerosa severa, ca. racterizada por manifestaciones sistémicas más acentuadas, dolor abdominal, anemia, hipoalbuminemia y fiebre, deben ser hospitalizados para reposición hidroelectrolítica y alimentación parenteral. Además de salazosulfapiridina se recomienda metilprednisolona endovenosa 1 a 2 $\mathrm{mg} \cdot \mathrm{kg} \cdot$ dja o hidrocortisona endovenosa 10 $\mathrm{mg} \cdot \mathrm{kg} \cdot$ día, además de corticoides en forma de enema. La mayoría de los pacientes responden a este tratamiento en un período de 15 dias, aunque algunos pueden tardar más ${ }^{9}$. En los casos que no responden hay que recurrir a la colectomía, ante el peligro de perforación o hemorragia digestiva masiva. El primero de los pacientes descritos sufrió una crisis severa que respondió a tratamiento médico. La mortalidad en este grupo es del $40 \%$ en los primeros cinco affos, falleciendo la mitad de los pacientes en e] primer ataque. Después de la primera crisis se. vera, los episodios recurrentes son de menor severidad. El segundo caso presentó una colitis ulcerosa leve que mejoro con el tratamiento y se ha mantenido en buenas condiciones hasta el momento. En general, el pronóstico de estos pacientes con crisis iniciales leves es bueno. $\mathbf{L a}$ colitis ulcerosa en etapa de remisión debe seguir siendo tratada con salazosulfapiridina, de esta manera se previenen las recaídas.

\section{Resumen}

En consideración a la baja incidencia y poca frecuencia con que se publican casos de colitis ulcerosa en riños de nuestro medio, se describe el cuadro clínico de una niña y un varón de 14 y 10 años, respectivamente. Ambos presentaron deposiciones diarreicas sanguinolentas, general. mente nocturnas, asociadas a dolor abdominal, pujo y tenesmo y deterioro severo del estado de nutrición. El estudio bacteriológico y parasito. lógico de deposiciones descartó infección. El điagnóstico se apoyó en la presencia de imágenes espiculares y pérdida de haustras del colon en el enema baritado; mucosa hiperémica, friable y erosiones en la vísión rectosigmoidoscópica e 
intensa inflamación, distorsión, abscesos y marcada disminución de las células caliciformes en las criptas de la mucosa en la histologia de las biopsias rectales. En ambos pacientes el tratamiento consistió en el uso de salazosulfapiridina, observándose una buena respuesta en ambos.

(Palabras claves: colitis ulcerosa, radiología, endoscopía, histopatología, diagnóstico, tratamiento.)

\section{Referencias}

I. Kirchner, B.; M.D. . [nflammatory bowel disease in children, Pediatr Cin North Am 1988; 35: 189205.

2. Silverman, $A_{\text {; }}$ Roy, C.: Pediatric Clinical Gastroenterology Lnflammatory bowel disease. pp. 349390. 3rd, edition, The CV Mosby Co., St. Louis 1983.

3. Zúfiga, A.; Rahmer, A.; Llanos, O. et al.: Colit is ulcerosa. Indicaciones y resultados del tratamiento quirúrgico. Rev Méd Chile 1987:115: 1155-1160.
4. Jacobelli, $S$.; Sepúiveda, $C$; Quintana, $C$. et at.: Manifestaciones articulares de la colitis ulcerosa: Experjencia con 88 pacientes. Rev Méd Chile 1984; 112: $797-801$.

5. Zúñiga, A.; Quintana, C.; Morales, C.; Ordóf̆ez, M.: Curso y pronóstico de la colitis ulceros extensa. Rev Méd Chile 1982;110:871876.

6. Peralta, O,i Orrego, $H$ : Algunos aspectos de la colitis ulcerosa idiopática en Chile. Comunicación preliminar del Directorio de la Sociedad Chilena de Gastroenterología. Rev Méd Chile 1956; 84; 343-349.

7. Donoso, A.; Amenábar, E.; Donkaster, R.; Godoy. M. Morales, 1 , Feres, $A$. : Contribución al estudio de la colitis ulcerosa inespecífica. Frecuencia general en Chile y aspectos clínicos y de laboratorio en 82 asos. Rev Méd Chile 1956; 84: 674-684.

8. Monning, A.P.; Bulgim, O.R.; Dixon, M.F.; Axan. A.T. Screening by colonoscopy for colonic epithelial dysplasia in inflammatory bowel disease. Gut 1987;28: $1489-1494$.

9. Rosenthal, S,; Snyder, J; Hendricks, K.; Wat. ker, $w$.: Growth failure and inflammatory bowel disease. Approach to treatment of a complicated adolescent problem. Pediatrics 1983; 72: $481-490$. 